\title{
Electrostatic Potential Mapping by Secondary-electron Voltage-contrast and Electron-beam-induced-current in TEM
}

\author{
Myung-Geun $\operatorname{Han}^{1}$, Joseph A. Garlow ${ }^{2}$, and Yimei Zhu ${ }^{1}$ \\ ${ }^{1 .}$ Condensed Matter Physics \& Materials Science, Brookhaven National Laboratory, Upton, NY, USA \\ 2. Materials Science and Engineering Department, Stony Brook University, Stony Brook, NY, USA
}

Nanoscale control of electric fields in modern electronic devices is a prerequisite to improving their functionalities to satisfy the scaling demand for high-density electronic devices. One of the key factors is interfaces/junctions, where built-in electric fields are generally predicted due to electronic band bending. Experimental measurements of these built-in electric fields are of great importance to predict and control charge transport properties in advanced devices that involve photovoltaic effects, rectifications, or capacitance modulations, etc. Electron holography has been an important tool for revealing electrostatic and magnetic field distributions in microelectronics and magnetic-based memory devices [1], however, its utility is hindered by several practical constraints, such as charging artifacts and limitations in sensitivity and in field of view. We report electron-beam-induced-current (EBIC) and secondaryelectron voltage-contrast (SE-VC) with an aberration-corrected electron probe in a transmission electron microscope (TEM), as complementary techniques to electron holography, to measure electric fields and surface potentials, respectively. These two techniques were applied to ferroelectric thin films, multiferroic nanowires, and single crystals [2]. A key advantage of EBIC and SE-VC with thin TEM samples is that the interaction volume is small due to the reduced electron beam broadening. In addition, electron-hole-pair recombination at the top and bottom surface regions in TEM samples is dramatically enhanced as compared with bulk as the electronic states at surfaces likely serve as recombination centers. We have used aberration-corrected JEOL ARM $200 \mathrm{CF}$ with the probe current density of $\sim 0.22$ $\mathrm{nA} / \mathrm{nm}^{2}$. The electrical connection to TEM samples for EBIC and SE-VC was made using a commercial TEM holder (Nanofactory Instruments).

Figure 1 shows EBIC and $\mathrm{SE}-\mathrm{VC}$ results obtained from $\mathrm{PbZr}_{0.2} \mathrm{Ti}_{0.8} \mathrm{O}_{3}$ (PZT) film grown on $\mathrm{Nb}$-doped $\mathrm{SrTiO}_{3}(\mathrm{Nb}-\mathrm{STO})$ substrate. EBIC/SE-VC images were taken simultaneously with HAADF images. The EBIC signal (bright contrast) is clearly seen at the interface of PZT/Nb-STO in the biased part of sample, while the EBIC signal was not observed for the closed-circuit configuration. Active SE-VC data obtained from the same sample show a systematic change in SE intensity with external biases applied to the $\mathrm{Nb}-\mathrm{STO}$ substrate. In Figure 2, we have performed $\mathrm{SE}-\mathrm{VC}$ on $\mathrm{YMn}_{2} \mathrm{O}_{5}$ and $\mathrm{YMnO}_{3}$ nanowires synthesized by the molten salt method. Both ends of the $\mathrm{YMn}_{2} \mathrm{O}_{5}$ nanowire are contacted - on one end to a $\mathrm{W}$ probe and the other end to the $\mathrm{Cu}$ grid using hydrocarbon contamination accumulating under prolonged electron beam irradiation. Then, $+/-10 \mathrm{~V}$ external biases were applied to the $\mathrm{Cu}$ gird with respect to the $\mathrm{W}$ probe. A variation in SE-VC contrast with external biases is shown in line profiles taken along the long axis of the nanowire. There is a clear trend of the potential dependence with the observed SE brightness, similar to what was observed for the PZT sample shown in Figure 1. Meantime, the atomic resolution SEM image of $\mathrm{YMnO}_{3}$ nanowire (Fig. 2e) indicates that the backscattered electron contribution is not negligible. This study demonstrates EBIC and SE-VC with aberration-corrected electron probes, which can be utilized to visualize electrostatic potentials around TEM samples [3].

References:

[1] M. R. McCartney, et al. Ultramicroscopy 110 (2010), p. 375. 
[2] M.-G. Han, et al, Ultramicroscopy 177 (2017), p. 14.

[3] This work was supported by the Materials Science and Engineering Divisions, Office of Basic Energy

Sciences of the U.S. Department of Energy under Contract No. DESC0012704. Authors are thankful to C. H. Ahn (Yale Univ.), S. S. Wong (Stony Brook Univ.), and S.-W. Cheong (Rutgers Univ.) for their samples.
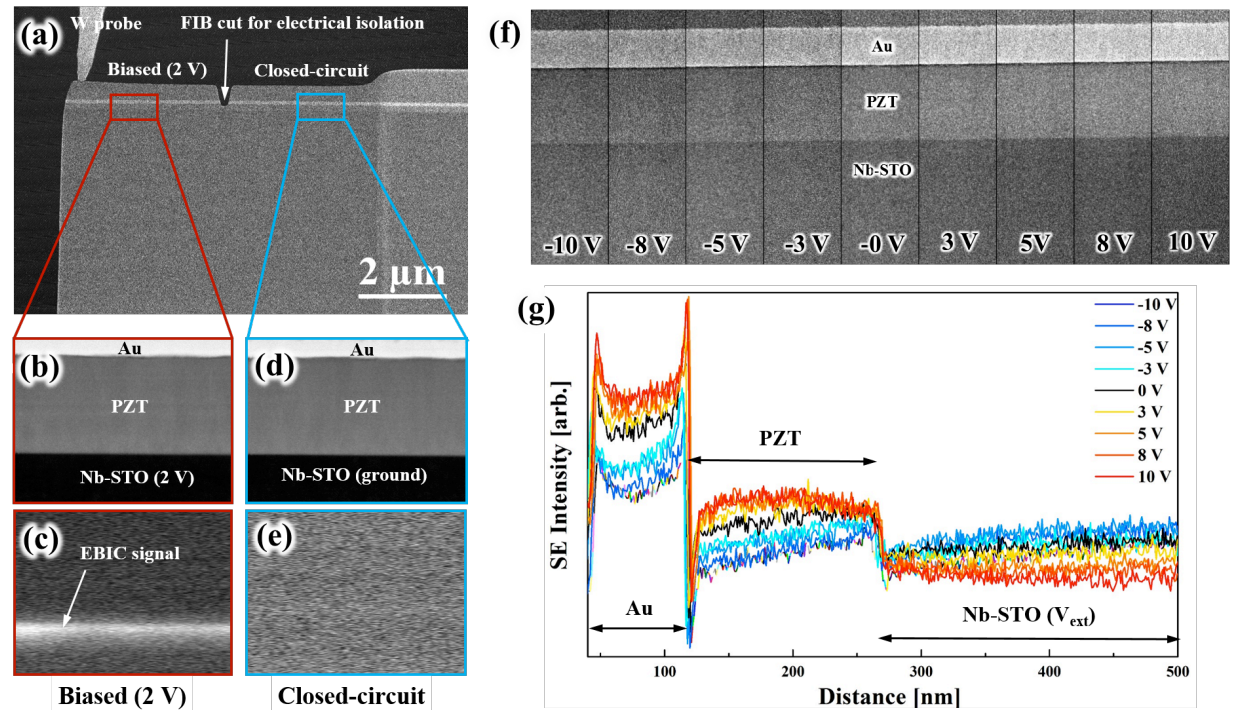

Figure 1. EBIC and active SE-VC measurements from $\mathrm{PbZr}_{0.2} \mathrm{Ti}_{0.8} \mathrm{O}_{3}$ (PZT) thin film grown on (001) Nb-doped $\mathrm{SrTiO}_{3}$ substrate. (a) STEM HAADF image showing an electrical contact made in a FIB-prepared TEM sample. (b) and (c) are HAADF and EBIC images, respectively, obtained from the biased section in (a). (d) and (e) are HAADF and EBIC images, obtained from an unbiased section shown in (a). (f) SEM images obtained with various external biases applied to the Nb-STO substrate. Note SE intensity varies systematically with external biasing. (g) Line profiles of SE intensity across $\mathrm{Au} / \mathrm{PZT} / \mathrm{Nb}-\mathrm{STO}$ interfaces.

(a)

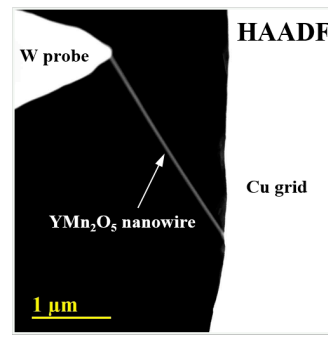

(c)

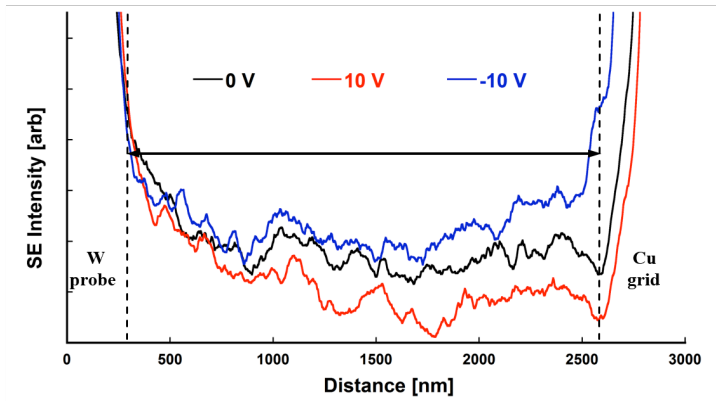

(b)

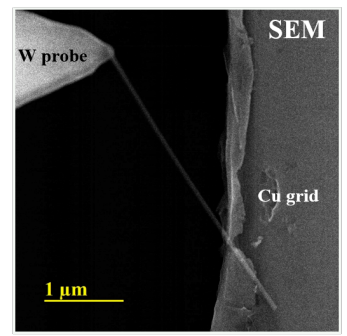

(e)
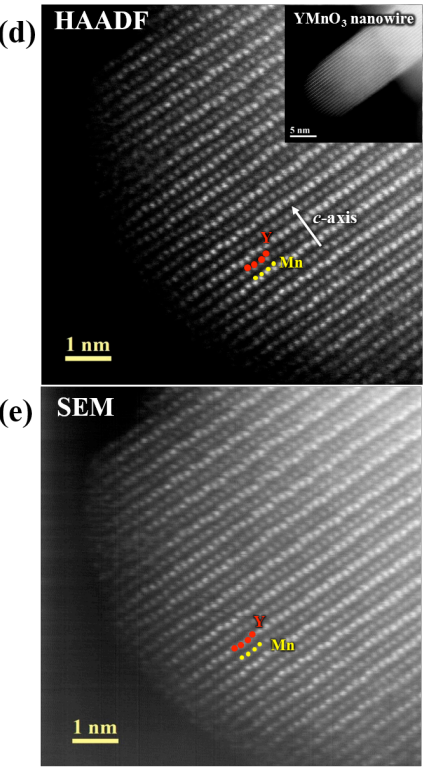

Figure 2. SE-VC of $\mathrm{YMn}_{2} \mathrm{O}_{5}$ and $\mathrm{YMnO}_{3}$. a) STEM HAADF and b) STEM SEM image of $\mathrm{YMn}_{2} \mathrm{O}_{5}$ nanowires electrically contacted by piezo-controlled $\mathrm{W}$ probe and $\mathrm{Cu}$ grid. c) Line profiles of SE-VC taken along the long axis of the $\mathrm{YMn}_{2} \mathrm{O}_{5}$ nanowire under $+/-10 \mathrm{~V}$ biases. (d) Atomic resolution STEM HAADF and (e) STEM SEM image of $\mathrm{YMnO}_{3}$ nanowire. Two images were acquired simultaneously. 